\title{
Estimating the Genetic Architecture of Fruit Quality Traits in Melon Using a Genomic Library of Near Isogenic Lines
}

\author{
Iban Eduardo ${ }^{1}$, Pere Arús, and Antonio José Monforte ${ }^{2}$ \\ Laboratori de Genètica Molecular Vegetal CSIC-IRTA, Departament Genètica Vegetal, Carretera de \\ Cabrils s/n, 08348 Cabrils (Barcelona), Spain \\ Javier Obando and Juan Pablo Fernández-Trujillo \\ Instituto de Biotecnología Vegetal y Departamento de Ingeniería de Alimentos y del Equipamiento \\ Agrícola, Universidad Politécnica de Cartagena, Campus Paseo Alfonso XIII, 48, ETSIA, 30203 \\ Cartagena (Murcia), Spain
}

Juan Antonio Martínez

Departamento de Producción Vegetal, Universidad Politécnica de Cartagena, Campus Paseo Alfonso XIII, 48, ETSIA, 30203 Cartagena (Murcia), Spain

\author{
Antonio Luís Alarcón \\ Departamento de Ciencia y Tecnología Agraria, Universidad Politécnica de Cartagena Campus, \\ Paseo Alfonso XIII, 48, ETSIA, 30203 Cartagena (Murcia), Spain \\ Jose María Álvarez \\ Unidad de Tecnología en Producción Vegetal, Centro de Investigación y Tecnología Agroalimentaria \\ de Aragón. Apartado 727, 50080 Zaragoza, Spain \\ Esther van der Knaap
Department of Horticulture and Crop Science, The Ohio State University/OARDC, Wooster, OH 44691
}

AdDitional INDEX words. Cucumis melo, introgression line, QTL, G $\times$ E interaction, heritability

Abstract. A melon (Cucumis melo L.) genomic library of near-isogenic lines derived from the cross between the Spanish cultivar Piel de Sapo and the exotic accession PI 161375 has been evaluated for fruit quality traits in four different locations. Traits evaluated were fruit weight, soluble solids content, maximum fruit diameter, fruit length, fruit shape index, ovary shape index, external color, and flesh color. Among these traits, soluble solids content showed the highest genotype $\times$ environment interaction, whereas genotype $\times$ environment interactions for fruit shape and fruit weight were low. Heritability was high for all traits except soluble solids content, with the highest value for fruit shape and ovary shape. Ten to $\mathbf{1 5}$ quantitative trait loci were detected for soluble solids content, fruit diameter, fruit length, and fruit shape; and four to five for ovary shape, external color, and flesh color. Depending on the trait, between $13 \%$ and $40 \%$ of the detected quantitative trait alleles from PI 161375 increased the trait, and between $60 \%$ and $87 \%$ of them decreased it, resulting in some PI 161375 alleles of interest for breeding. Most of the quantitative trait loci detected in previous experiments could be verified with the near-isogenic line population. Future studies with the melon near-isogenic line genomic library will provide a better understanding of the genetic control of melon fruit quality in a wider context related to agronomy, genetics, genomics and metabolomics studies.

Received for publication 11 Apr. 2006. Accepted for publication 28 Sept. 2006. This work was funded in part by grants AGL2003-09175-C02-01 and AGL2003-09175-C02-02 from the Spanish Ministry of Education and Science and Fondo Europeo de Desarrollo Regional (FEDER, European Union), and the Fundación Séneca de la Región de Murcia (00620/PI/04). A.J.M. was partly supported by a contract from Instituto Nacional de Investigación y Tecnología Agraria y Alimentaria (INIA) and by a fellowship from the Departament d'Universitats, Recerca i Societat de la Informació (Generalitat de Catalunya, Spain). I.E. and J.O. were supported by fellowships from the Spanish Ministry of Education and Science and the Spanish Ministry of Foreign Affairs respectively.

We thank Angel Montejo, Antonio Ortigosa, Eduard Moreno, Fuensanta García, Esperanza Truque, Maria Carmen García-Abellán, Mónica Cánovas (CIFACITA S.L.), Manuela Selva, Ana Belén Pérez, and Nick Welty for technical assistance. We also thank Semillas Fitó S.A. for providing the seeds of the parental PS line.

${ }^{1}$ Current address: Institute of Plant Biotechnology, University of Stellenbosch,

Merriman Avenue, 7602 Stellenbosch, South Africa.

${ }^{2}$ Corresponding author. E-mail: antonio.monforte@irta.es.
The use of wild or exotic germplasm as a source for novel allelic variation has been proposed as a strategy to recover at least part of the genetic variability lost during domestication (Ladizinsky, 1998). Exotic germplasm has been used to search for resistance genes or new alleles with favorable effects on fruit quality traits to be transferred to elite germplasm (Fernie et al., 2006; McCouch, 2004; Tanksley and McCouch, 1997; Zamir, 2001). However, some disease resistance pathways and most of the fruit quality traits are complex and controlled by several quantitative trait loci (QTL), making an efficient selection of favorable alleles difficult.

The development of molecular marker saturated maps allowed the thorough analysis of QTLs using different population structures (Tanksley, 1993) as well as the development of populations suitable for QTL analysis, such as introgression lines and near-isogenic lines (NILs). A NIL genomic library 
consists of a set of lines, each containing a single homozygous chromosome segment from a donor parent in the genetic background of an elite cultivar, all together providing complete coverage of the donor parent genome. Analysis of NIL genomic libraries significantly facilitates QTL analysis. These populations give better estimates of individual QTL effects (Eshed and Zamir, 1995; Tanksley et al., 1996), QTL $\times$ environment, QTL $\times$ genetic background, and QTL $\times$ QTL interactions (Eshed and Zamir, 1996; Monforte et al., 2001). Furthermore, after QTLs are isolated in NILs, they can be combined in an elite cultivar to specific traits such as those for fruit morphology (Tanksley, 2004) or yield (Gur and Zamir, 2004). NILs have been used for the study of different aspects of biology, including fruit sugar content (Fridman et al., 2004), fruit shape (FS) and size (reviewed in Tanksley, 2004), fruit vitamin content (Rousseaux et al., 2005), leaf dissection (Holtan and Hake, 2003), and metabolic profiling (Overy et al., 2005). Potentially, any interesting measurable trait can be studied and dissected using NIL populations.

The family Cucurbitaceae includes economically important species such as cucumber (Cucumis sativus L.), squash (Cucurbita maxima Duch.), zucchini (C. pepo L.), watermelon [Citrullus lanatus (Thunb.) Matsum. and Nakai] and melon ( $C$. melo). C. melo is the most diversified species of the genus Cucumis L., and this variability is reflected at the morphological, physiological, biochemical (Burger et al., 2003; Kirkbride, 1993; Liu et al., 2004; Miccolis and Saltveit, 1991; Whitaker and Davis, 1962), and molecular (Akashi et al., 2002; Mliki et al., 2001; Monforte et al., 2003; Stepansky et al., 1999) levels. A high proportion of the genetic variability can be found in African, Indian and Oriental germplasm, which are considered exotic germplasm relative to European and North American cultivars (Akashi et al., 2002; Mliki et al., 2001; Monforte et al., 2003; Stepansky et al., 1999).

Even though 162 major genes controlling different aspects of melon biology have been described (Pitrat, 2002), most of them have not been mapped. In addition, the genetic inheritance of most important agronomic traits is largely unknown, due, at least in part, to the fact that full linkage maps have only recently become available (Gonzalo et al., 2005; Oliver et al., 2001; Périn et al., 2002a). QTLs involved in fruit quality traits have been detected in three different crosses involving European cultivars and exotic Asian accessions (Monforte et al., 2004; Périn et al., 2002b). New experiments are still needed to assess if these alleles from exotic germplasm can be included into modern melon breeding programs. To obtain good estimates of the effects of QTLs involved in fruit quality traits, we present the phenotypic analysis of fruit quality traits using a melon NIL genomic library unique in the family Cucurbitaceae. This library consists of a set of lines with an introgression from the exotic Korean melon accession PI 161375 in the Spanish melon cultivar Piel de Sapo background, and covers the complete genome of this exotic accession (Eduardo et al., 2005).

\section{Materials and Methods}

Plant material. A set of 27 NILs was derived from a cross between two $C$. melo genotypes: 'Piel de Sapo' (PS) and the exotic Korean accession 'Shongwan Charmi' [SC (PI 161375)]. The NILs were developed by marker-assisted selection after several generations of backcrossing and selfing, as described by
Eduardo et al. (2005). Most of them contained a single homozygote introgression from SC, with only three of them containing a single heterozygote introgression as shown in Fig. 1. The introgressions represented in this set of 27 NILs together cover most of the SC genome.

EXPERIMENTAL DESIGN AND PHENOTYPIC EVAluation. The NILs were evaluated in four locations in the summer of 2004: Cabrils (Spain), Zaragoza (Spain), Cartagena (Spain), and Wooster (Ohio). In Cabrils, 10 plants of each NIL and 100 controls (PS) were grown in a greenhouse in peat bags, drip irrigated, with $0.25-\mathrm{m}$ spacing between plants. The greenhouse was divided in five blocks and an equal number of plants for each NIL and controls were randomized within each block. Plants were hand pollinated, ensuring only one fruit per plant. In Zaragoza, four plots of three plants for each NIL and 14 plots of PS were randomized in the field. The flowers were open pollinated, and three to five fruit from each plot were harvested. In Cartagena and Wooster, 10 plants of each NIL and 50 or 55 controls, respectively, were transferred to the field in a completely randomized design. The flowers were open pollinated. In Cartagena, the plantation had a grid with a distance of $2 \mathrm{~m}$ between rows and $1.4 \mathrm{~m}$ within hills $\left(0.36\right.$ plants $\left./ \mathrm{m}^{2}\right)$.

The agronomic evaluation included the following traits: fruit weight (FRW) in grams; soluble solids content (SSC) measured with a hand refractometer from homogenized melon flesh or juice obtained with a metal juicer (in the case of Cartagena); maximum fruit diameter (FD) and fruit length (FL) in centimeters; FS index as the ratio between FL and FD; ovary shape index (OS) as the ratio between maximum ovary diameter and ovary length, only in Cabrils; and external color (ECOL) evaluated subjectively in vivo or using digital pictures as green, dark green, or light green, and flesh color (FCOL) as white, green, light green, and orange. All traits were measured in all locations except for OS, which was studied only in Cabrils. Additional observations of plants and fruit (plant structure, plant health, fruit external spot pattern, necrosis of fruit placental tissue) were recorded when the phenotypes were very different from the PS control.

DATA ANALYSIS. All statistical analyses were performed with JMP (version 5.1.2 for Windows; SAS Institute, Cary, N.C.).

The interaction of the effects of the SC introgressions with the different environments was studied only in Cabrils, Zaragoza, and Cartagena, because it was not possible to obtain data from all the lines in Wooster as a result of unfavorable weather conditions. Only NILs that set fruit an all three previously cited locations were included in the analysis.

The model for the two-way analysis of variance (ANOVA) was

$$
Y_{i j k}=\mu+G_{i}+E_{j}+G_{i j}+e_{i j k}
$$

where $\mu$ represents the population mean value; $G_{i}$, the effect of the genotype [NIL (fixed effect)]; $\mathrm{E}_{\mathrm{j}}$, the environment effect [Cabrils, Zaragoza, or Cartagena (random effect)]; $\mathrm{GE}_{\mathrm{ij}}$, the genotype $\times$ environment $(\mathrm{G} \times \mathrm{E})$ interaction effect; and $\mathrm{e}_{\mathrm{ijk}}$, the error or random residual effect. Significance of the effects was studied by an $F$ test and variance components were also estimated. NILs with a higher $\mathrm{G} \times \mathrm{E}$ interaction were identified by comparing the NIL mean estimates, according to the model, and the actual NIL means, by a $t$ test.

Heritability $\left(\mathrm{h}^{2}\right)$ was estimated in each location independently from the ANOVA: 


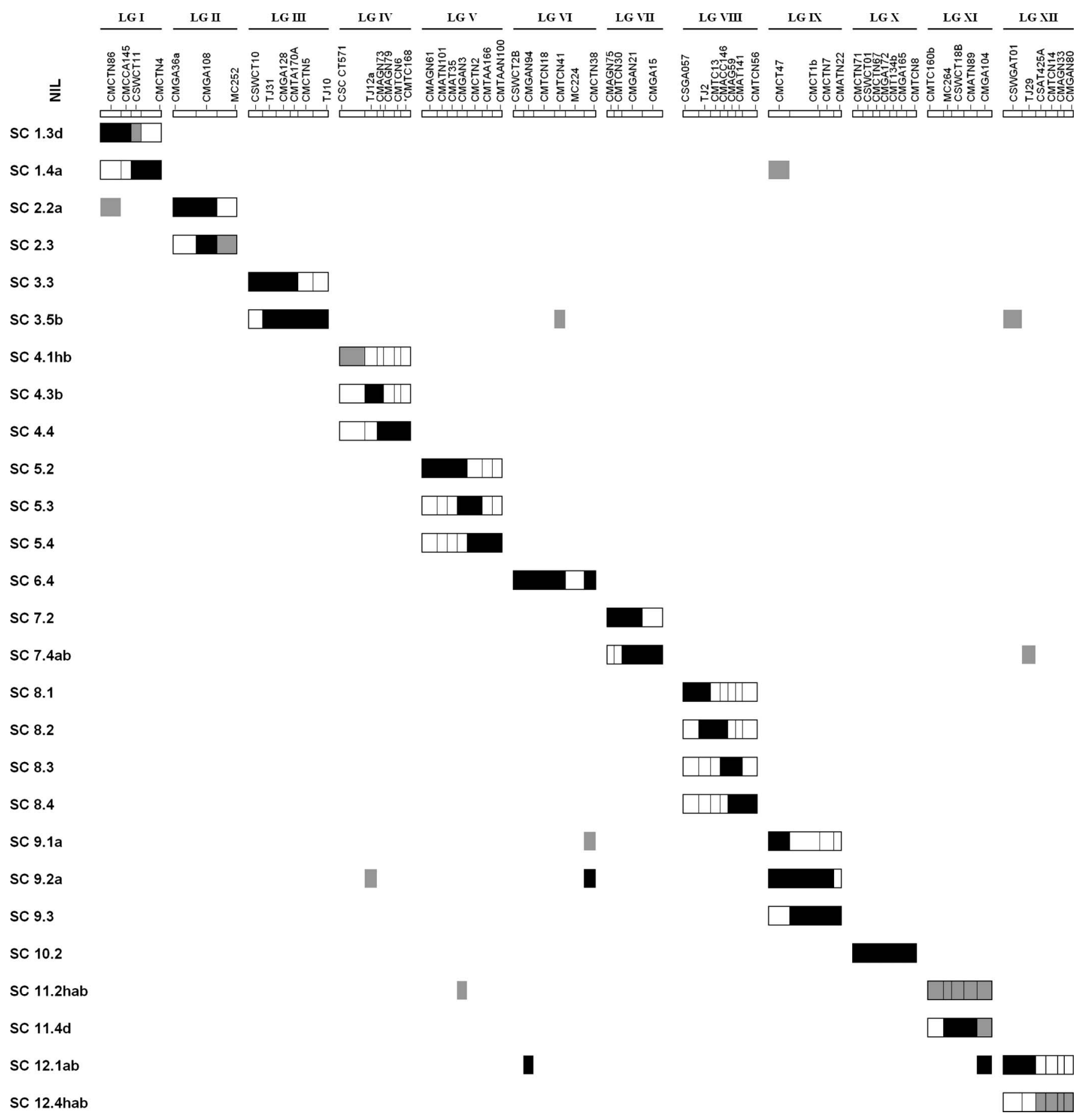

Fig. 1. Graphical genotypes of the Cucumis melo NILs evaluated. The lines are shown on the right. a, more than one introgression; $b$, the NIL has an introgression slightly different from the introgression of the NIL with the same number described in Eduardo et al. (2005), although it covers the same genomic region; $h$, the introgression is heterozygous; and d, that part of the introgression is heterozygous. Linkage groups (LG) are depicted at the top. LG nomenclature is according to Périn et al. (2002a) and the molecular map is according to Gonzalo et al. (2005). Heterozygous and homozygous introgressions from SC are shown in gray and black respectively.

$$
\mathrm{Y}_{\mathrm{ij}}=\mu+\mathrm{NIL}_{\mathrm{i}}+\mathrm{e}_{\mathrm{ij}}
$$

where NIL $_{i}$ is the effect of the NIL, and the estimate of $h^{2}$ was calculated as

$$
\mathrm{h}^{2}=\mathrm{V}_{\mathrm{b}} / \mathrm{V}_{\mathrm{t}}
$$

where $\mathrm{V}_{\mathrm{b}}$ and $\mathrm{V}_{\mathrm{t}}$ are the between-family (genetic variance) and total variances respectively estimated from the mean squares of the ANOVA. The statistical significance of $\mathrm{h}^{2}$ was obtained from the previous ANOVA.

Pearson correlation coefficients between traits were calculated independently in each location. Correlations were considered significant at $P<0.005(0.005=0.05 / 10$ correlation pairs per location), except in Cabrils, where the level of significance was fixed at $P<0.003(0.003=0.05 / 15$ correlations performed in Cabrils). 
To study the effect of the SC introgressions, NIL mean values were compared with the mean of the control genotype PS using the Dunnet contrast (Dunnet, 1955) with type I error $\alpha \leq$ 0.05 for each location.

The number of QTLs was estimated assuming there was only one QTL per introgression, and when two NILs with overlapping introgressions showed significant effects, the QTL was located in the overlapping region.

\section{Results}

Phenotypic analysis of the control genotype. Trait means and SDS for PS in different locations are given in Table 1. PS fruit are large and oval, with high SSC, white flesh, green skin, and a sparse spot pattern. The more evident differences between locations were for FRW (very low in Zaragoza) and SSC (very low in Wooster). These differences can be explained by the weather conditions. In Zaragoza it was dry, which is associated with small fruit. The weather in Wooster was very humid, often overcast, and with low solar irradiation. Those weather conditions affect negatively melon vegetative growing, probably reducing leaf carbon assimilation, limiting the source for fruit sugar accumulation (Long et al., 2004; Pardossi et al., 2004).

GENOTYPE $\times$ ENVIRONMENT INTERACTION, HERITABILITY, AND TRAIT CORRELATIONS. Genetic and environment effect components and their interactions were significant for all traits (Table 2), although the contribution of each component to the variation was remarkably different between traits. FL and FS showed a low environment effect $(24 \%$ and $22 \%$ of total variance respectively) and a low $\mathrm{G} \times \mathrm{E}$ interaction effect $(4.5 \%$ and $9.5 \%$ of total variance respectively), FD and FRW showed a high environment effect $(54 \%$ and $46 \%)$ but a low $\mathrm{G} \times \mathrm{E}$ interaction effect $(6.3 \%$ and $5.4 \%)$, and SSC showed an important environment effect $(21.8 \%)$ and $\mathrm{G} \times \mathrm{E}$ interaction effect $(24.1 \%)$.

Heritability estimates are given in Table 3 . Heritability was highly significant for all traits in every location. The highest values were obtained for FS and OS: 0.62 and 0.84 respectively. The lowest values were found for SSC (range, 0.18-0.51), with intermediate values for FD, FL, and FRW (range, 0.29-0.72).

Correlations between traits are shown in Table 4. In all locations, the highest correlations were found between FRW and FD (mean correlation, 0.89), and between FL and FS (mean correlation, 0.74). The correlation between FS and OS was also quite strong in Cabrils. SSC correlated less significantly with other traits, and these correlations were not consistent between locations.

Phenotypic analysis of The NIL population fOR FRUIT QUALITY TRAITS. NIL trait means with respect to the PS control mean in each location, and the statistical significance of the comparisons with the control for SSC, FRW, FD, FL, and FS are depicted in Fig. 2. Data from NIL SC5-4 were not obtained in any location because of germination problems. The remainder of the NILs were evaluated in Cartagena and Zaragoza. In Cabrils, NILs SC1-4a and SC5-2 did not produce fruit because the plants suffered severe necrosis. NILs SC6-4, SC8-4, SC10-2, and SC11-2hab did not produce fruit in Wooster because most of these plants were affected by disease. There was wide variation for every trait, including phenotypic values that were up to $50 \%$ higher than the PS control.

Soluble SOLIDS CONTENT. Nineteen NILs were significantly different from the control in at least one location. Two of them had fruit with between a $15 \%$ and $22 \%$ higher SSC than the control (SC1-4a, SC3-5ab) and the other 16 had between $11 \%$ and $31 \%$ less SSC (SC2-2a, SC2-3d, SC3-3, SC4-4, SC5-2, SC6-4, SC7-2, SC7-4ab, SC8-2, SC8-3, SC8-4, SC9-1a, SC9-3, SC11-2hab, SC12-1ab, SC12-4hb). NIL SC10-2 had significantly opposite allelic effects: higher SSC in Zaragoza and lower in Cartagena. The most consistent NILs were SC2-3d (reducing between $18 \%$ and $31 \%$ ), SC3-5ab (increasing between $16 \%$ and $19 \%$ ), and SC8-4 (decreasing $13 \%$ and $16 \%$ ), with significant effects in two locations. The rest of the NILs (except for SC10-2) showed significant effects only in one location. The pairs of NILs SC2-2a and SC2-3d, and SC7-2 and SC7-4ab shared a fragment of the introgression, so each pair of NILs may represent a single QTL. NILs SC8-2, SC8-3, and SC8-4 shared different regions of linkage group (LG) VIII; the minimum number of QTLs represented in these NILs was two. Finally, the estimated minimum number of QTLs affecting this trait was 15.

Fruit weight. Fourteen NILs were significantly different from the control in at least one location. Four of them had an increase in FRW between $16 \%$ and $39 \%$ (SC1-4a, SC6-4, SC9-3, $\mathrm{SC} 11-2 \mathrm{hab})$, and the other 10 had a decrease in FRW between 20\% and 56\% (SC3-3, SC3-5ab, SC4-3b, SC4-4, SC5-2, SC7-4ab, SC8-1, SC8-2, SC8-4, SC10-2). The most consistent NILs were SC4-3b (decreasing between 54\% and 56\%), SC4-4 (decreasing between $22 \%$ and $41 \%$ ), and SC6-4 (increasing between $23 \%$ and $29 \%$ ), with significant differences in all locations where they were evaluated, and SC8-1, which gave significant differences in three of them decreasing between $32 \%$ and $36 \%$. SC7-4ab, SC8-2, SC8-3, SC11-2hab, and SC12-4hb were the most divergent among locations $(P<0.05)$. SC8-3 and SC12-4hb did not show significant effects in any location, but showed significant different effects among locations. This result may indicate that the putative QTLs represented in those NILs have relatively strong interaction effects but low main effects. The pairs of NILs SC3-3 and SC3-5ab, SC4-3b and SC4-4, and SC8-1 and SC8-2 shared part of their introgressions, so the

Table 1. Means and standard deviations for Cucumis melo cultivar Piel de Sapo (PS) in different locations for the fruit quality traits evaluated in the trials: FRW, SSC, FD, FL, FS, and OS.

\begin{tabular}{lccrrrrr}
\hline Location & $\mathrm{N}$ & FRW $(\mathrm{g})$ & SSC $(\%)$ & FD $(\mathrm{cm})$ & FL $(\mathrm{cm})$ & FS & OS \\
\hline Cabrils & 52 & $2056.20 \pm 432.58$ & $10.40 \pm 1.30$ & $14.59 \pm 0.97$ & $18.41 \pm 1.84$ & $1.26 \pm 0.08$ & $1.79 \pm 0.15$ \\
Cartagena & 47 & $2013.82 \pm 300.01$ & $11.98 \pm 1.89$ & $13.71 \pm 0.81$ & $19.07 \pm 1.57$ & $1.39 \pm 0.10$ & y \\
Wooster & 14 & $1748.14 \pm 735.49$ & $8.69 \pm 2.62$ & $13.28 \pm 1.77$ & $18.84 \pm 2.81$ & $1.42 \pm 0.08$ & $\mathrm{y}$ \\
Zaragoza & 15 & $1144.72 \pm 223.23$ & $11.03 \pm 1.08$ & $11.46 \pm 0.85$ & $16.89 \pm 1.36$ & $1.48 \pm 0.06$ & $\mathrm{y}$ \\
\hline
\end{tabular}

${ }^{\mathrm{z} N u m b e r}$ of plants.

yata not available. 
Table 2. Partition of the variance in genetic $(G)$ and environmental $(E)$ components, their interaction $(G \times E)$ and residual error including data from three locations: Cabrils, Cartagena, and Zaragoza, Spain.

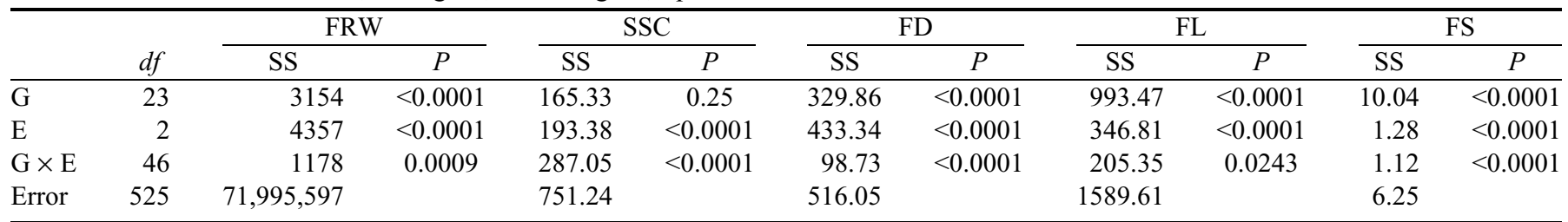

The sum of squares (SS) and the level of significance of the corresponding $F$ test ( $P$ value) are given for the evaluated Cucumis melo traits: fruit weight, soluble solids content, fruit diameter, fruit length, and fruit shape. NILs SC1.4a, SC4-3b, SC5.2, and SC5.4 were not included because these NILs did not set fruit in at least one location.

minimum number of QTLs represented in this set of NILs was three. The estimated minimum number of QTLs affecting this trait was 11 .

Fruit Diameter. Thirteen NILs were significantly different in at least one location. For four of them, the maximum FD increased by between 7\% and 17\% (SC6-4, SC9-3, SC11-2hab, $\mathrm{SC} 12-4 \mathrm{hb}$ ) and for the other nine it decreased by between $8 \%$ and 23\% (SC4-3b, SC4-4, SC5-2, SC7-4ab, SC8-1, SC8-2, SC8-3, SC8-4, SC10-2). The most consistent NILs were SC4-3b (decreasing between $21 \%$ and 23\%), SC6-4 (increasing between $11 \%$ and 17\%), and SC8-2 (decreasing between 10\% and $23 \%$ ), with significant effects in all locations for which data were available. NILs SC7-4ab, SC8-1, SC8-3, SC8-4, SC9-3, and SC12-4hb showed significant different effects among locations. SC1-3d did not have significant effects in any location, but showed significant different effects among locations. The pair of NILs SC4-3b and SC4-4 shared part of their introgressions; the minimum number of QTLs represented in this set of NILs was one. NILs SC8-1, SC8-2, SC8-3, and SC8-4 shared different parts of LG VIII, representing at least two QTLs. The estimated minimum number of QTLs was 10.

Fruit Length. Thirteen NIL means were significantly different in at least in one location. For three of them, FL increased by between $10 \%$ and $29 \%$ (SC1-4a, SC8-3, SC11$2 \mathrm{hab}$ ) and for the other 10 it decreased by between $11 \%$ and 29\% (SC1-3d, SC3-5ab, SC4-3b, SC4-4, SC6-4, SC7-4ab, SC8-1, SC8-3, SC11-4d, SC12-1ab). The most consistent NILs were SC4-3b (decreasing between 22\% and 28\%), SC4-4 (decreasing between $18 \%$ and 29\%), SC8-1 (decreasing between 12\% and 17\%), and SC8-3 (increasing between 16\% and $29 \%$ ), with significant effects in all locations for which data were available. NILs SC1-3d and SC7-4ab had the most significant variation between locations. SC8-2, SC9-3, and SC 10-2 did not have significant effects in any location, but showed significant different effects among locations. The pair of NILs SC4-3b and SC4-4 shared part of their introgressions; the minimum number of QTLs represented in this set of NILs was one. The estimated minimum number of QTLs was 11.

Table 3. Heritability shown by the Cucumis melo traits: FRW, SSC, FD, FL, FS, and OS evaluated in the four locations.

\begin{tabular}{lcccccc}
\hline Location & FRW & SSC & FD & FL & FS & OS \\
\hline Cabrils & 0.19 & 0.35 & 0.31 & 0.29 & 0.62 & 0.62 \\
Cartagena & 0.60 & 0.18 & 0.71 & 0.59 & 0.72 & z \\
Wooster & 0.36 & NA & 0.39 & 0.55 & 0.70 & z \\
Zaragoza & 0.49 & 0.51 & 0.45 & 0.72 & 0.84 & z \\
\hline
\end{tabular}

${ }^{\mathrm{z}}$ Data not available.

NA, not applicable.
Fruit ShaPe. Sixteen NIL means were significantly different in at least one location. Eight of them had fruit that were between $11 \%$ and $52 \%$ more elongated than the control (SC1-4a, SC7-2, SC8-2, SC8-3, SC8-4, SC9-1a, SC10-2, SC11-2hab) and the other eight produced fruit that were between $10 \%$ and 28\% rounder (SC1-3d, SC2-2a, SC3-5ab, SC4-4, SC6-4, SC8-1, SC11-4d, SC12-1ab). NILs SC6-4 (decreasing between 22\% and 26\%), SC8-2 (increasing between $21 \%$ and $36 \%$ ), and SC8-3 (increasing between $22 \%$ and $52 \%$ ) showed significant effects in all locations. $\mathrm{G} \times \mathrm{E}$ interaction was stronger for NILs SC2-2a, SC8-1, SC8-3, and SC11-4d. NIL SC9-1a did not have significant effects in any location, but showed significant different effects among locations. SC8-2 and SC8-3 shared part of their introgression, so it was assumed that only one QTL was represented in both NILs. The minimum number of QTLs estimated as affecting this trait was 15 .

Ovary SHAPE. This trait was evaluated only in Cabrils. Six NILs showed significant effects, three with $19 \%$ to $29 \%$ more elongated ovaries than the control (SC8-2, SC8-3, SC10-2) and the other three with $10 \%$ to $22 \%$ rounder ovaries (SC4-4, SC64, SC12-1ab). SC8-2 and SC8-3 shared part of their introgression, so we assumed that only one QTL was represented in both NILs. The minimum number of QTLs estimated affecting this trait was five.

Flesh COLOR. Most NILs had a white FCOL, the same as for PS. NILs SC1-3d and SC8-3 had green flesh, NILs SC7-2 and SC7-4ab had light-green flesh, and some fruit of NIL SC3-5ab had orange flesh (Table 5). These effects were the same in all locations. SC7-2 and SC7-4ab shared their introgressions, so we assumed that these NILs include only one QTL. The estimated minimum number of QTLs affecting this trait was four.

EXTERNAL COLOR. PS and most NILs were classified as green ECOL. NILs SC3-5ab, SC8-3, and SC8-4 had a light-green ECOL, whereas NILs SC7-2, SC7-4ab, and SC10-2 had darkgreen ECOL (Table 5). The effects on ECOL were the same in all locations. The NIL pairs SC8-3 and SC8-4, and SC7-2 and SC7-4ab shared their introgressions, so we assumed that these NILs include a minimum number of two QTLs. The minimum number of QTLs affecting this trait was four.

OTHER TRAITS. Differences in other agronomic and fruit quality traits were observed, although no extensive analysis was conducted. In Cabrils, NILs SC1-4a and SC5-2 had severe leaf necrosis and no fruit was harvested. In Cartagena and Cabrils, there was some necrosis of fruit placental tissue in NILs SC2-3d and SC9-1a. Also, in both locations, the skin of fruit from NILs SC7-2 and SC7-4ab had a highly dense spot pattern, whereas NILs SC3-5ab and SC6-4 had a sparse spot pattern.

Overall, between 4 and 15 QTLs were detected for several important fruit traits, and $51 \%$ of them were detected in at least 
Table 4. Correlations among Cucumis melo traits: FRW, SSC, FD, FL, and FS.

\begin{tabular}{|c|c|c|c|c|c|c|c|c|c|c|}
\hline \multicolumn{6}{|c|}{ Cabrils } & \multicolumn{5}{|c|}{ Wooster } \\
\hline & FRW & $\mathrm{SSC}$ & FL & FD & FS & & FRW & $\mathrm{SSC}$ & FL & FD \\
\hline$\overline{\mathrm{SSC}}$ & -0.28 & & & & & SSC & 0.41 & & & \\
\hline FL & 0.55 & $-0.68^{*}$ & & & & FL & $0.64 *$ & 0.05 & & \\
\hline FD & $0.93 *$ & -0.05 & 0.27 & & & FD & $0.89 *$ & 0.45 & 0.25 & \\
\hline FS & -0.14 & -0.60 & $0.74^{*}$ & -0.45 & & FS & -0.03 & -0.29 & $0.74^{*}$ & -0.46 \\
\hline OS & -0.24 & -0.46 & $0.61 *$ & -0.48 & $0.91 *$ & & & & & \\
\hline \multicolumn{6}{|c|}{ Cartagena } & \multicolumn{5}{|c|}{ Zaragoza } \\
\hline & FRW & $\mathrm{SSC}$ & FL & FD & & & FRW & $\mathrm{SSC}$ & FL & FD \\
\hline $\mathrm{SSC}$ & -0.06 & & & & & $\mathrm{SSC}$ & 0.20 & & & \\
\hline FL & $0.64 *$ & -0.09 & & & & FL & $0.65^{*}$ & 0.23 & & \\
\hline FD & $0.93 *$ & -0.08 & 0.36 & & & FD & $0.86^{*}$ & 0.04 & 0.29 & \\
\hline FS & -0.25 & -0.06 & 0.55 & $-0.57 *$ & & FS & 0.17 & 0.20 & $0.84 *$ & -0.6 \\
\hline
\end{tabular}

${ }^{*} P<0.005$, except in Cabrils, which indicates $P<0.003$.

two locations. For all traits, increasing trait alleles from the exotic source SC were identified.

\section{Discussion}

GENETIC DETERMINATION OF FRUIT QUALITY TRAITS IN MELON. The analysis of $\mathrm{G} \times \mathrm{E}$ interaction, heritability, and trait correlations provides an overview of the genetic control of the traits under study (Tables 2, 3, and 4). The genetic component was highly significant between and within locations for all traits, confirming the high genetic variability between PS and PI 161375, and this genetic variability could be used for breeding purposes. Large differences were observed in the weight of the genetic component on the total variance. FS showed the highest heritability, the highest genetic component between locations, and low $\mathrm{G} \times \mathrm{E}$ interaction, which fits with the previous hypothesis that predicts the highly heritable, polygenic control of FS in melon (Monforte et al., 2004; Périn et al., 2002b). The low environmental effect on FS may be explained because melon FS is determined preanthesis, during early flower development (Table 4) (Monforte et al., 2005; Périn et al., 2002b), as it is has been frequently observed in tomato [Solanum lycopersicum L. (Tanksley, 2004)]. That early determination could limit the environmental effects that might affect this trait. FRW showed a lower heritability, but also a low $\mathrm{G} \times \mathrm{E}$ interaction, indicating that even though the effect of the
SSC

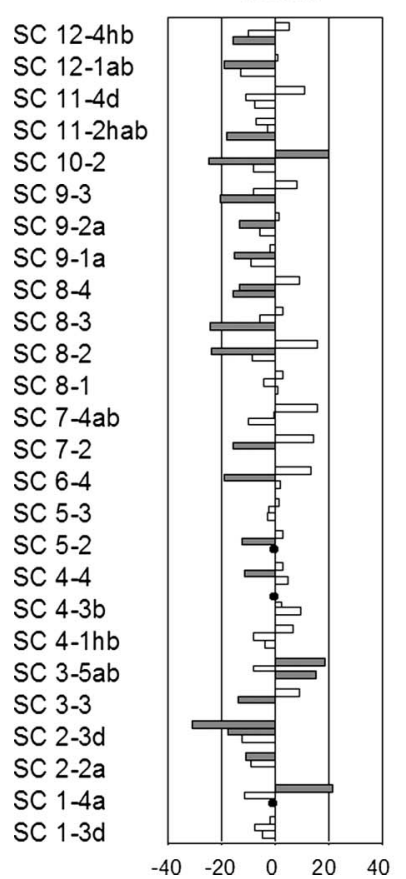

FW

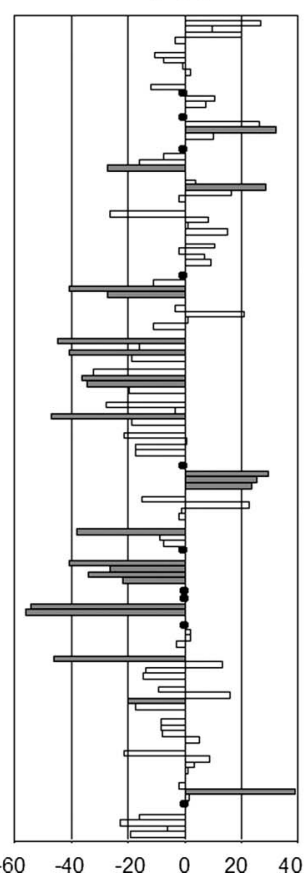

FD

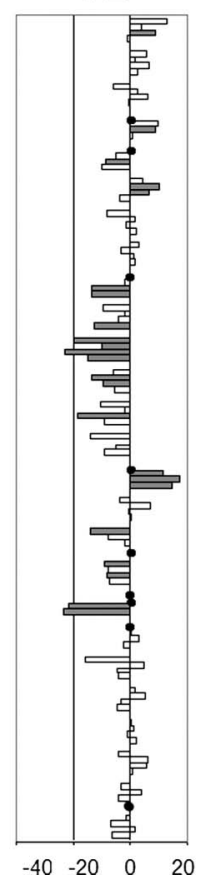

FL

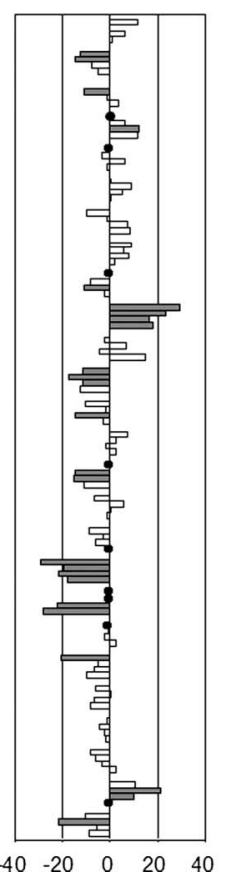

FS

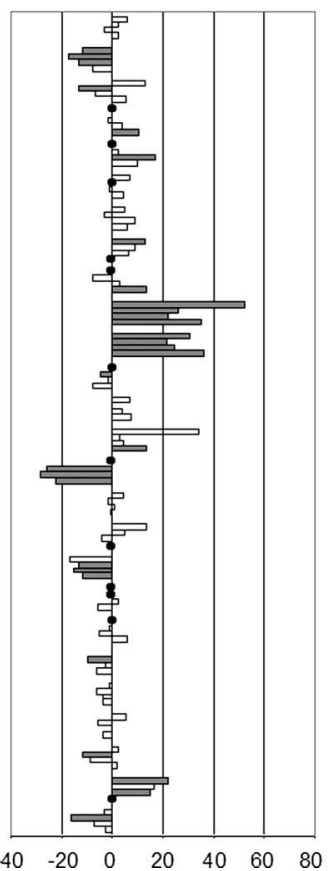

os

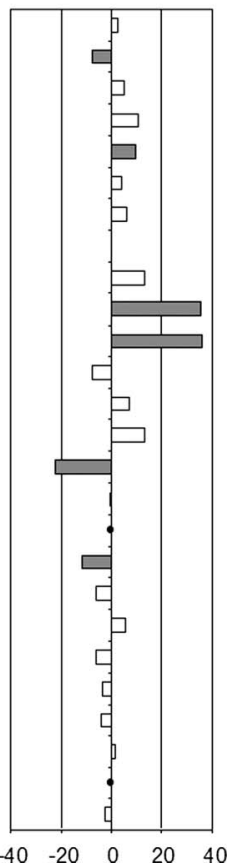

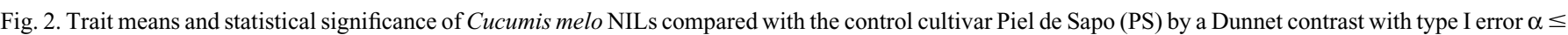
0.05 at each location. NIL names are shown on the right, traits are indicated above, and the percentage difference with respect to the control PS is shown below the graphics. Gray and white bars indicate whether differences are significant or nonsignificant respectively. Each trait for each line is represented by four bars, indicating the data from the four different locations (top to bottom: Wooster, Ohio; Zaragoza, Spain; Cartagena, Spain; Cabrils, Spain). • indicates no data available. Data for SSC was obtained only in Zaragoza, Cartagena, and Cabrils. Data for OS was obtained only in Cabrils. NIL SC5.4 is not included because it was not possible to obtain fruit from this NIL in any location. 
Table 5. Classification of Cucumis melo NILs displaying FCOL and ECOL different from the 'Piel de Sapo' control genotype.

\begin{tabular}{llllll}
\hline & Flesh color & & & \multicolumn{2}{c}{ External color } \\
\hline Green & Light green & Orange & & Light green & Dark green \\
\hline SC1-3d & SC7-2 & SC3-5b & & SC3-5b & SC7-2 \\
SC8-3 & SC7-4ab & & & SC8-3 & SC7-4ab \\
& & & SC8-4 & SC10-2 \\
\hline
\end{tabular}

environment on the variance of this trait is important, genetic effects are consistent in different environments. In contrast, $\mathrm{SSC}$ values had the lowest heritability and a high $\mathrm{G} \times \mathrm{E}$ interaction, as has been observed in melon (Monforte et al., 2004) and other species, such as peach [Prunus persica (L.) Batsch (LiBin et al., 2004)], mango [Mangifera indica L. (Brettell et al., 2004)], kiwifruit [Actinidia deliciosa (A. Chev.) C.F. Liang and A.R. Ferguson (Marsh et al., 2003)], chili [Capsicum annuum L. (Doshi, 2003)], and cucumber (Dijkhuizen and Staub, 2002). SSC involves complex metabolic processes, whereas the previous traits are related to morphology. Sugar accumulation needs coordination of the sugar metabolism pathway in the fruit sink. A large number of environmental factors may influence sugar accumulation (e.g., temperature, radiation, and nutrition), and it occurs approximately during the last 2 weeks of fruit development (Gao et al., 2004). Environmental events occurring before sugar accumulation begins can mean this process follows a different ontogeny. Furthermore, differences in the stage of maturity of the fruit can also produce high variability within genotypes (Paris et al., 2003). This last factor is especially important in nonclimacteric cultivars (such as the PS and the NILs studied here), being difficult to determine the maturity stage before harvest.

The highest correlations observed in all locations were between FD and FRW, and FL and FS. The high correlation between FL and FS has been reported for genetically unrelated melon lines (Monforte et al., 2005; Périn et al., 2002b), although it is not common in tomato. For example, the correlation is significant but low in Solanum pennelli Correl introgression lines (Eshed and Zamir, 1995; Zamir et al., 2006), and it was not significant in a cross between wild and cultivated tomatoes (Lippman and Tanksley, 2001). In contrast, in the latter reports, the significant correlation between FS and FD was more common, whereas, in the current population, the correlation between these traits is much lower than between FL and FS. This apparently greater effect of FL on FS in melon compared with tomato suggests that the determination of FS in melon may involve different processes.

No strong correlation was observed between SSC and any other trait, as observed previously (Monforte et al., 2004; Taha et al., 2003). One important consequence of this is the possibility of manipulating FS and FRW to fulfill market or consumer demands without decreasing such a highly important fruit quality parameter as sugar content.

EFFICIENCY OF THE MELON GENOMIC LIBRARY OF NILS FOR DISSECTING COMPLEX TRAITS. Because of the limited number of markers available in melon, some genomic regions were not covered during the NIL development (Eduardo et al., 2005). This implies that a QTL assigned to a genomic region according to the NIL graphic genotype might actually be located in an undetected introgression. Some of the NILs used in the current report have known additional introgressions (Fig. 1). To minimize this potential problem, we have interpreted that NILs with different graphic genotype according to Fig. 1, and a phenotype significantly different from the control PS, harbor different QTLs. Thus, the most likely position of a QTL would be the target introgression of the NIL. Currently, we are developing new markers and we will control them in NIL populations to verify their genomic composition. Meanwhile, we will focus the discussion on QTL number and allelic effects.

QTLs involved in melon fruit quality traits have been previously reported using different mapping populations, such as $\mathrm{F}_{2}$, double haploid lines (DHLs) (Monforte et al., 2004), and recombinant inbred lines (Périn et al., 2002b). With the analysis of the genomic library of NILs, a larger number of QTLs for all traits has been detected, demonstrating the utility of the current NIL population for dissecting genetically complex traits in melon. For example, 15 potential QTLs for FS and SSC have been detected using the NILs, whereas Périn et al. (2002b) detected seven QTLs for FS, and Monforte et al. (2004) detected seven QTLs for FS and five for SSC. All the QTLs detected for SSC, FRW, and FS by Monforte et al. (2004) have been detected in at least one location with the NIL population. For example, QTLs $f_{S} 1.1, f_{s} 3.1, f_{S} 5.1, f_{s} 6.1, f_{S} 6.2, f_{S} 7.1, f_{S} 9.1$, and $f_{S} 11.1$ (Monforte et al., 2004) are represented in a introgressions of NILs SC8-3, SC7-2, SC11-4d, SC1-3d, SC1-4a, SC9-2a, SC10-2, and SC12-1ab respectively, with the predicted phenotype as shown in Fig. 2. [The differences in QTL and NIL numbers are the result of the originally different linkage group nomenclature between the maps of Gonzalo et al. (2005) and Périn et al., (2002a).]

A complete set of 57 NILs with a unique homozygote introgression from SC, covering most of the SC genome, and with a mapping resolution higher than the 27 lines used in this work, are currently available (Eduardo et al., 2005) and could be useful to detect more QTLs or to obtain better estimates for them.

Fruit traits are usually controlled by a large number of QTLs (Grandillo et al., 1999). Tanksley (2004) suggested that most of the variability for fruit size and shape in tomato could be incited by a few major QTLs. A number of candidates for major QTLs in melon fruit variability can be described, because of their highly repeatable effects on analysis of the melon $\mathrm{F}_{2}$, DHL, and NIL populations. Good candidates for FRW are the QTLs included in NILs SC6-4 and SC11-2hab that were also detected by Monforte et al. (2004); and NILs SC4-4 and SC8-1, which affected FRW consistently in all the environments. Six candidates for major QTLs for FS are NILs SC1-3d and SC1-4a, SC8-2, SC8-3, SC11-2hab and SC11-4d, and SC12-1ab, which were also detected by Monforte et al. (2004) and Périn et al. (2002b), and the one corresponding to NIL SC6-4. For SSC, only three QTLs have been detected in most environments. These QTLs were also detected by Monforte et al. (2004), and they correspond to NILs SC2-3d, SC3-5ab, and SC8-3. Repeatability of effects of these QTLs across environments and populations is especially striking because of the low heritability and high $\mathrm{G} \times \mathrm{E}$ interaction observed for this trait (discussed earlier). There is also the QTL on NIL SC2-3d that was detected in most NIL trials, as shown in Fig. 2, and most of the previous trials with $\mathrm{F}_{2}$ and DHL populations (Monforte et al., 2004).

FRW and FS can be divided into several trait components. The analysis of some detailed mathematical descriptors for shape attributes has contributed to the better understanding of the genetic basis of FS observed in some extremely elongated tomato cultivars (Brewer et al., 2006; Van der Knaap and 
Tanksley, 2003; Van der Knaap et al., 2002). In the current report, we only studied simple components such as FL and FD. Generally, a highly significant correlation was found between FRW and FD, and between FL and FS (described earlier). Consequently, the differences in FRW for some NILs could be attributed mainly to differences in FD (NILs SC5-2, SC6-4, SC8-2, SC9-3, SC10-2), and variation in FS could be attributed mainly to variation in FL (SC1-3d, SC1-4a, SC8-3, SC11-4d, $\mathrm{SC} 12-1 \mathrm{ab})$. Therefore, two genetically independent processes determining fruit morphology can be defined: longitudinal elongation mainly determining FS, and equatorial growth mainly determining FRW. A third process can be also defined, causing variation in both FL and FD, and consequently in FRW, but no change in FS (SC4-3b, SC7-4ab, SC8-1). This putative third process would be involved in the control of the global fruit size, without any preference for developmental axis.

It has been proposed that melon FCOL is controlled by two genes that interact epistatically: green flesh [ $g f$ (Hughes, 1948)] and white flesh [wf (Iman et al., 1972)]. Monforte et al. (2004) mapped $g f$ at the bottom of LG VIII and proposed that, in the current cross, orange flesh color would be under different genetic control, involving several loci. The analysis of the melon NIL genomic library confirms that $g f$ maps on the bottom of LG VIII, in the overlapping regions of NILs SC8-3 and SC8-4. Other subtle effects on flesh color were also detected in NILs SC1-3d (green flesh), SC3-5ab (orange flesh), and SC7-2 and SC7-4ab (light-green flesh). These results suggest that melon flesh color could be controlled by major genes and by genes causing minor effects.

The genetic control of ECOL is not well understood. Monforte et al. (2004) proposed that ECOL was controlled by several loci with epistatic interactions. Four loci were described: ecol3.1, ecol7.1, ecol9.1, and ecol10.1. Two of these (ecol3.1 and ecol9.1) were confirmed with the NILs SC7-2, SC7-4ab, and SC10-2. Furthermore NILs SC3-5ab, SC8-3, and SC8-4 had a light-green ECOL. In the case of SC8-3 and SC8-4, the different ECOL could be the result of a pleiotropic effect of $g f$. These results confirm the complex genetic control of ECOL in the current cross.

A striking observation was the severe leaf necrosis in NILs SC1-4a and SC5-2, only in Cabrils. The most likely explanation is that these NILs are susceptible to sulfur (S) dusting, as sulfur was used, only in Cabrils, to control powdery mildew [Podosphaera xanthii (Castagne) Braun and Shichkoff]. These NILs have been grown in Cabrils, in S-free greenhouse conditions, without signs of leaf necrosis. Perchepied et al. (2004) found that $\mathrm{S}$ susceptibility is common in melon accessions. The genetic control of this trait is polygenic, with a major QTL (Sr) giving most of the variance, and several minor QTLs. Perchepied et al. (2004) mapped $\mathrm{Sr}$ on LG I, the same genomic region of the introgression contained in NIL SC1-4a. SC1-4a suffered more severe leaf necrosis than PI 161375, indicating interaction of $\mathrm{Sr}$ with the PS genetic background. NIL SC5-2 may carry another QTL for S susceptibility not detected previously.

The results presented here demonstrate the high versatility of the current genomic library of NILs for studying the genetics of complex traits in melon. This population was very powerful for dissecting quantitative traits, qualitative traits under complex control, such as flesh and external color, and for simplifying the analysis of traits that need relatively complex measurement, such as color intensity (ECOL and FCOL), necrosis of fruit placental tissue, spot pattern, and leaf necrosis associated with $\mathrm{S}$ dusting.

IMPLICATIONS FOR THE FUTURE. The melon NIL genomic library has revealed alleles from PI 161375 that could be very useful for breeding and dissecting the genetic control of fruit traits. Further analysis of this NIL population, including fine mapping, QTL interactions, candidate gene analysis, and mapbased cloning, will provide a body of data comparable with other model species. The integration of current advances in fruit development analysis in model species (Frary et al., 2000; Liu et al., 2002; Tanksley, 2004; Van der Knaap et al., 2004) with experiments involving the melon NIL genomic library, will also increase knowledge of the genetic control of melon fruit quality traits.

Melon germplasm has impressive fruit phenotype variability (Monforte et al., 2005; Stepansky et al., 1999). The allelic variability detected in this NIL population is only a small portion of the melon genetic variability. The analysis of other melon NIL populations derived from crosses involving distantly related genotypes would identify the QTLs responsible of the overall fruit variability observed within this species.

The melon NILs could also be a resource for modern breeding programs. For example, FS and FRW are traits of high interest for the agrifood chain. These traits are critical in reducing mechanical damage and transport costs as well as for designing cultivars appropriate for automatic fruit processing, increasing the added value for the melon grower. Moreover, these traits are also important in consumer preferences (Olías, 1995). In the current work, some highly heritable QTLs modifying FS and FRW have been identified, which would allow the design of melons adapted for different markets (seed companies, growers, food processors, retailers, final markets, or consumers) by introducing these QTLs in modern cultivars, using marker-assisted selection. Furthermore, Kader (2002) suggested that fruit and vegetable biotechnology-oriented studies must focus on postharvest quality traits of interest to consumers, the market, and the industry, including appearance, texture, flavor and aroma, nutritional quality, physiological disorders, pathogen susceptibility, and food safety. The melon NIL genomic library could also be useful for dissecting most of these traits.

In summary, we present a study of the genetics of fruit quality in melon using a newly available melon NIL genomic library. The results presented here can provide a starting point for further characterization of the genetic factors involved in melon fruit quality. Future studies with this and other similar populations will allow efficient, systematic, and straightforward manipulation of the allelic variability present in melon germplasm.

\section{Literature Cited}

Akashi, Y., N. Fukuda, T. Wako, M. Masuda, and K. Kato. 2002. Genetic variation and phylogenetic relationships in east and south Asia melons, Cucumis melo L., based on the analysis of five isozymes. Euphytica 125:385-396.

Brettell, R.I.S., P.R. Johnson, V.J. Kulkarni, W. Muller, and I.S.E. Bally. 2004. Inheritance of fruit characters in hybrid mangoes produced through controlled pollination. Acta Hort. 645:319-326.

Brewer, M.T., L. Lang, K. Fujimura, N. Dujmovic, S. Gray, and E. van der Knaap. 2006. Development of a controlled vocabulary and software application to analyze fruit shape variation in tomato and other plant species. Plant Physiol. 141:15-25. 
Burger, Y., U. Sa'ar, A. Distelfeld, N. Katzir, Y. Yeselson, A. Shen, and A.A. Schaffer. 2003. Development of sweet melon (Cucumis melo L.) genotypes combining high sucrose and organic acid content. J. Amer. Soc. Hort. Sci. 128:537-540.

Dijkhuizen, A. and J.E. Staub. 2002. QTL conditioning yield and fruit quality traits in cucumber (Cucumis sativus L.): Effects of environment and genetic background. J. New Seeds 4:1-30.

Doshi, K.M. 2003. Genetic architecture of chili (Capsicum annuum L.). Capsicum Eggplant Nwsl. 22:33-36.

Dunnet, C.W. 1955. A multiple comparison procedure for comparing several treatments with a control. J. Amer. Stat. Assoc. 50:10961121.

Eduardo, I., P. Arus, and A.J. Monforte. 2005. Development of a genomic library of near isogenic lines (NILs) in melon (Cucumis melo L.) from the exotic accession PI 161375. Theor. Appl. Genet. 112:139-148.

Eshed, Y. and D. Zamir. 1995. An introgression line population of Lycopersicon pennellii in the cultivated tomato enables the identification and fine mapping of yield-associated QTL. Genetics 141:1147-1162.

Eshed, Y. and D. Zamir. 1996. Less-than-additive epistatic interactions of quantitative trait loci in tomato. Genetics 143:1807-1817.

Fernie, A.R., Y. Tadmor, and D. Zamir. 2006. Natural genetic variation for improving crop quality. Curr. Opin. Plant Biol. 9:196-202.

Frary, A., T.C. Nesbitt, S. Grandillo, E. van der Knaap, B. Cong, J. Liu, J. Meller, R. Elber, K.B. Alpert, and S.D. Tanksley. 2000. fw2.2: A quantitative trait locus key to the evolution of tomato fruit size. Science 289:85-88.

Fridman, E., F. Carrari, Y.S. Liu, A.R. Fernie, and D. Zamir. 2004. Zooming in on a quantitative trait for tomato yield using interspecific introgressions. Science 305:1786-1789.

Gao, Z., M. Petreikov, Y. Burger, S. Sehn, and A.A. Schaffer. 2004. Stachyose to sucrose metabolism in sweet melon (Cucumis melo) fruit mesocarp during the sucrose accumulation stage, p. 471-475. In: A. Lebeda and H.S. Paris (eds.). Progress in cucurbit genetics breeding research. Palacký University in Olomouc, Olomouc, Czech Republic.

Gonzalo, M.J., M. Oliver, J. Garcia-Mas, A. Monfort, R. DolcetSanjuan, N. Katzir, P. Arus, and A.J. Monforte. 2005. Simplesequence repeat markers used in merging linkage maps of melon (Cucumis melo L.). Theor. Appl. Genet. 110:802-811.

Grandillo, S., H.M. Ku, and S.D. Tanksley. 1999. Identifying the loci responsible for natural variation in fruit size and shape in tomato. Theor. Appl. Genet. 99:978-987.

Gur, A. and D. Zamir. 2004. Unused natural variation can lift yield barriers in plant breeding. Public Library Sci. Biol. 2:e245.

Holtan, H.E.E. and S. Hake. 2003. Quantitative trait locus analysis of leaf dissection in tomato using Lycopersicon pennellii segmental introgression lines. Genetics 165:1541-1550.

Hughes, M.B. 1948. The inheritance of two characters of Cucumis melo and their interrelationship. Proc. Amer. Soc. Hort. Sci. 52:399-402.

Iman, M.K., M.A. Abo-Bakr, and H.Y. Hanna. 1972. Inheritance of some economic characters in crosses between sweet melon and snake cucumber. Inheritance of qualitative characters. J. Agr. Sci. 3:363380.

Kader, A.A. 2002. Opportunities in using biotechnology to maintain postharvest quality and safety of fresh produce. HortScience 37:467468 .

Kirkbride, J.H. 1993. Biosystematic monograph of the genus Cucumis (Cucurbitaceae). Pakway, Boone, N.C.

Ladizinsky, G. 1998. Plant evolution under domestication. Kluwer Academic Publishers, Dordrecht, the Netherlands.

LiBin, Z., X. Xiao, W. XueDong, and D. Gang. 2004. Variability and inheritance of some characters in self-crossed progeny of Okubo peach cultivar. J. Fruit Sci. 21:308-310.

Lippman, Z. and S.D. Tanksley. 2001. Dissecting the genetic pathway to extreme fruit size in tomato using a cross between the small-fruited wild species Lycopersicon pimpinellifolium and L. esculentum var. Giant Heirloom. Genetics 158:413-422.

Liu, J., J. van Eck, B. Cong, and S.D. Tanksley. 2002. A new class of regulatory genes underlying the cause of pear-shaped tomato fruit. Proc. Natl. Acad. Sci. USA 99:13302-13306.

Liu, L., F. Kakihara, and M. Kato. 2004. Characterization of six varieties of Cucumis melo L. based on morphological and physiological characters, including shelf-life of fruit. Euphytica 135:305313.

Long, R.L., K.B. Walsh, G. Rogers, and D.J. Midmore. 2004. Sourcesink manipulation to increase melon (Cucumis melo L.) fruit biomass and soluble sugar content. Austral. J. Agr. Res. 55:1241-1251.

Marsh, H.D., T. Paterson, A.G. Seal, and M.A. McNeilage. 2003. Heritability estimates in kiwifruit. Acta Hort. 622:221-229.

McCouch, S. 2004. Diversifying selection in plant breeding. Public Library Sci. Biol. 2:e347.

Miccolis, V. and M.E. Saltveit. 1991. Morphological and physiological changes during fruit growth and maturation of seven melon cultivars. J. Amer. Soc. Hort. Sci. 116:1025-1029.

Mliki, A., J.E. Staub, Z.Y. Sun, and A. Ghorbel. 2001. Genetic diversity in melon (Cucumis melo L.): An evaluation of African germplasm. Genet. Res. Crop Evol. 48:587-597.

Monforte, A.J., E. Friedman, D. Zamir, and S.D. Tanksley. 2001. Comparison of a set of allelic QTL-NILs for chromosome 4 of tomato: Deductions about natural variation and implications for germplasm utilization. Theor. Appl. Genet. 102:572-590.

Monforte, A.J., J. Garcia-Mas, and P. Arus. 2003. Genetic variability in melon based on microsatellite variation. Plant Breed. 122:153-157.

Monforte, A.J., M. Oliver, M.J. Gonzalo, J.M. Alvarez, R. DolcetSanjuan, and P. Arus. 2004. Identification of quantitative trait loci involved in fruit quality traits in melon (Cucumis melo L.). Theor. Appl. Genet. 108:750-758.

Monforte, A.J., I. Eduardo, S. Abad, and P. Arus. 2005. Inheritance mode of fruit traits in melon. Heterosis for fruit shape and its correlation with genetic distance. Euphytica 144:31-38.

Olías, J.M. 1995. Postrecolección de frutas y hortalizas: Ciencia o mercadotecnia? Fronteras de la Ciencia y la Tecnología 7:26-28.

Oliver, M., J. Garcia-Mas, M. Cardus, N. Pueyo, A.L. Lopez-Sese, M. Arroyo, H. Gomez-Paniagua, P. Arus, and M.C. de Vicente. 2001. Construction of a reference linkage map for melon. Genome 44:836845.

Overy, S.A., H.J. Walker, S. Malone, T.P. Howard, C.J. Baxter, L.J. Sweetlove, S.A. Hill, and W.P. Quick. 2005. Application of metabolite profiling to the identification of traits in a population of tomato introgression lines. J. Expt. Bot. 56:287-296.

Pardossi, L., P. Giacomet, F. Malorgio, F.M. Albini, C. Murelli, G. Serra, P. Vernieri, and F. Tognoni. 2004. The influence of growing season on fruit yield and quality of greenhouse melon (Cucumis melo L.) grown in nutrient film technique in a Mediterranean climate. J. Hort. Sci. Biotechnol. 75:488-493.

Paris, M., J.E. Staub, and J.D. McCreight. 2003. Determination of fruit sampling location for quality measurements in melon (Cucumis melo L.). Cucurbit Genet. Coop. Rep. 26:12-17.

Perchepied, L., C. Périn, C. Giovinazzo, D. Besombes, C. Dogimont, and M. Pitrat. 2004. Susceptibility to sulfur dusting and inheritance in melon, p. 353-357. In: A. Lebeda and H.S. Paris (eds.). Progress in cucurbit genetics breeding research. Palacký University in Olomouc, Olomouc, Czech Republic.

Périn, C., L.S. Hagen, V. De Conto, N. Katzir, Y. Danin-Poleg, V. Portnoy, S. Baudracco-Arnas, J. Chadoeuf, C. Dogimont, and M. Pitrat. 2002a. A reference map of Cucumis melo L. based on two recombinant inbred line populations. Theor. Appl. Genet. 104:10171034.

Périn, C., L.S. Hagen, N. Giovinazzo, D. Besombes, C. Dogimont, and M. Pitrat. 2002b. Genetic control of fruit shape acts prior to anthesis in melon (Cucumis melo L.). Mol. Genet. Genomics 266:933-941.

Pitrat, M. 2002. Melon gene list. 15 May 2006. <www.umresearch. umd.edu/CGC/genelist/melon.pdf $>$. 
Rousseaux, M.C., C.M. Jones, D. Adams, R. Chetelat, A. Bennett, and A. Powel. 2005. QTL analysis of fruit antioxidants in tomato using Lycopersicon pennellii introgression lines. Theor. Appl. Genet. 111:1396-1408.

Stepansky, A., I. Kovalski, and R. Perl-Treves. 1999. Intraspecific classification of melons (Cucumis melo L.) in view of their phenotypic and molecular variation. Plant Syst. Evol. 217: 313-332.

Taha, M., K. Omara, and A. El Jack. 2003. Correlation among growth, yield and quality characters in Cucumis melo L. Cucurbit Genet. Coop. Rep. 26:9-11.

Tanksley, S.D. 1993. Mapping polygenes. Annu. Rev. Genet. 27:205233.

Tanksley, S.D. 2004. The genetic, developmental, and molecular bases of fruit size and shape variation in tomato. Plant Cell 16(Suppl.):181189.

Tanksley, S.D., S. Grandillo, T.M. Fulton, D. Zamir, T. Eshed, V. Petiard, J. Lopez, and T. Beck-Bunn. 1996. Advanced backcross QTL analysis in a cross between an elite processing line of tomato and its wild relative L. pimpinellifolium. Theor. Appl. Genet. 92: 213-224.
Tanksley, S.D. and S.R. McCouch. 1997. Seed banks and molecular maps: Unlocking genetic potential from the wild. Science 277:10631066.

Van der Knaap, E., Z.B. Lippman, and S.D. Tanksley. 2002. Extremely elongated tomato fruit controlled by four quantitative trait loci with epistatic interactions. Theor. Appl. Genet. 104:241-247.

Van der Knaap, E., A. Sanyal, S.A. Jackson, and S.D. Tanksley. 2004. High-resolution fine mapping and fluorescence in situ hybridization analysis of sun, a locus controlling tomato fruit shape, reveals a region of the tomato genome prone to DNA rearrangements. Genetics 168:2127-2140.

Van der Knaap, E. and S.D. Tanksley. 2003. The making of a bell pepper-shaped tomato fruit: Identification of loci controlling fruit morphology in 'Yellow Stuffer' tomato. Theor. Appl. Genet. 107:139-147.

Whitaker, T.W. and G.N. Davis. 1962. Cucurbits, botany, cultivation and utilization. Interscience, New York.

Zamir, D. 2001. Improving plant breeding with exotic genetic libraries. Nat. Rev. Genet. 2:983-989.

Zamir, D., M. Causse, I. Paran, and F. Friedman. 2006. Real time QTL. 15 May 2006. <http://zamir.sgn.cornell.edu/Qt1/Html/home.htm>. 